\title{
Minimal and Complete Explanations for Critical Multi-attribute Decisions
}

\author{
Christophe Labreuche ${ }^{1}$ and Nicolas Maudet ${ }^{2}$ and Wassila Ouerdane ${ }^{3}$ \\ 1 Thales Research \& Technology \\ 91767 Palaiseau Cedex, France \\ christophe.labreuche@thalesgroup.com \\ 2 LAMSADE, Université Paris-Dauphine \\ Paris 75775 Cedex 16, France \\ maudet@lamsade.dauphine.fr \\ 3 Ecole Centrale de Paris \\ Chatenay Malabry, France \\ wassila.ouerdane@ecp.fr
}

\begin{abstract}
The ability to provide explanations along with recommended decisions to the user is a key feature of decision-aiding tools. We address the question of providing minimal and complete explanations, a problem relevant in critical situations where the stakes are very high. More specifically, we are after explanations with minimal cost supporting the fact that a choice is the weighted Condorcet winner in a multi-attribute problem. We introduce different languages for explanation, and investigate the problem of producing minimal explanations with such languages.
\end{abstract}

\section{Introduction}

The ability to provide explanations along with recommended decisions to the user is a key feature of decision-aiding tools [1,2]. Early work on expert systems already identified it as one of the main challenge to be addressed [3], and the recent works on recommender systems face the same issue, see e.g. [4]. Roughly speaking, the aim is to increase the user's acceptance of the recommended choice, by providing supporting evidence that this choice is justified.

One of the difficulties of this question lies on the fact that the relevant concept of an explanation may be different, depending on the problem at hand and on the targeted audience. The objectives of the explanations provided by an online recommender system are not necessarily the same as the ones of a pedagogical tool. To better situate our approach, we emphasize two important distinctive dimensions:

- data vs. process - following [5], we first distinguish explanations that are based on the data and explanations that are based on the process. Explanations based on the data typically focus on a "relevant" subset of the available data, whereas those based on the process make explicit (part of) the mathematical model underlying the decision. 
- complete vs. incomplete explanations - as opposed to incomplete explanations, complete explanations support the decision unambiguously, they can be seen as proofs supporting the claim that the recommended decision is indeed the best one. This is the case for instance in critical situations (e.g. involving safety) where the stakes are very high.

In this paper we shall concentrate on complete explanations based on the data, in the context of decisions involving multiple attributes from which, associating a preference model, we obtain criteria upon which options can be compared. Specifically, we investigate the problem of providing simple but complete explanations to the fact that a given option is a weighted Condorcet winner (WCW). An option is a WCW if it beats any other options in pairwise comparison, considering the relative weights of the different criteria. Unfortunately, a WCW may not necessarily exists. We focus on this case because (i) when a WCW exists it is the unique and uncontroversial decision to be taken, (ii) when it does not many decision models can be seen as "approximating" it, and (iii) the so-called outranking methods (based on the Condorcet method) are widely used in multi-criteria decision aiding, (iv) even though the decision itself is simple, providing a minimal explanation may not be.

In this paper we assume that the problem involves two types of preferential information (PI): preferential information regarding the importance of the criteria, and preferential information regarding the ranking of the different options.

To get an intuitive understanding of the problem, consider the following example.

Example 1. There are 6 options $\{a, b, c, d, e, f\}$ and 5 criteria $\{1, \cdots, 5\}$ with respective weights as indicated in the following table. The (full) orderings of options must be read from top (first rank) to bottom (last rank).

\begin{tabular}{|c|ccccc|}
\hline criteria & 1 & 2 & 3 & 4 & 5 \\
\hline weights & 0.32 & 0.22 & 0.20 & 0.13 & 0.13 \\
\hline ranking & $c$ & $b$ & $f$ & $d$ & $e$ \\
& $a$ & $a$ & $e$ & $f$ & $b$ \\
& $e$ & $f$ & $a$ & $b$ & $d$ \\
& $d$ & $e$ & $c$ & $a$ & $f$ \\
& $b$ & $d$ & $d$ & $c$ & $a$ \\
& $f$ & $c$ & $b$ & $e$ & $c$ \\
\hline
\end{tabular}

In this example, the WCW is $a$. However this option does not come out as an obvious winner, hence the need for an explanation. Of course a possible explanation is always to explicitly exhibit the computations of every comparison, but even for moderate number of options this may be tedious. Thus, we are seeking explanations that are minimal, in a sense that we shall define precisely below. What is crucial at this point is to see that such a notion will of course be dependent on the language that we have at our disposal to produce explanations. A tentative "natural" explanation would be as follows:

"First consider criteria 1 and 2, $a$ is ranked higher than $e, d$, and $f$ in both, so is certainly better. Then, $a$ is preferred over $b$ on criteria 1 and 
3 (which is almost as important as criterion 2). Finally, it is true that $c$ is better than $a$ on the most important criteria, but $a$ is better than $c$

on all the other criteria, which together are more important."

The aim of this paper is not to produce such natural language explanation, but to provide the theoretical background upon which such explanations can later be generated.

This abstract example may be instantiated in the following situations. In the first one, a decision-maker presents a choice recommendation regarding a massive investment before funding agency. The decision was based on a multi-criteria analysis during which criteria and preferences were elicited. In the second one, a committee (where members have different voting weights) just proceeded to a vote on a critical issue, and the chairman is now to explain why a given option was chosen as a result. The reason why we take these two concrete examples is that beyond their obvious similarity (members of the committee play the role of the criteria in the funding example), they share the necessity to produce a complete explanation. The type of explanation we seek for is relevant when the voters (for the committee example) are not anonymous, which is often the case in committee.

The remainder of this paper is as follows. In the next section, we provide the necessary background notions, and introduce in particular the languages we shall use for formulating explanations. Section 3 defines minimal complete explanations. Section 4 and Section 5 deal with languages allowing to express the preferences on the rankings of options only, starting with the language allowing basic statements, then discussing a more refined language allowing to "factor" statements. Finally, Section 6 discusses connections to related works, in particular argumentation theory.

\section{Background and Basic Definitions}

\subsection{Description of the choice problem}

We assume a finite set of options $O$, and a finite set of criteria $H=\{1,2, \ldots, m\}$. The options in $O$ are compared thanks to a weighted majority model based on some preferential information (PI) composed of preferences and weights. Preferences are linear orders, that is, complete rankings of the options in $O$, and $a \succ_{i} b$ stands for the fact that $a$ is strictly preferred over $b$ on criterion $i$. Weights are assigned to criteria, and $W_{i}$ stands for the weight of criterion $i$. Furthermore, they are normalized in the sense that they sum up to 1 . An instance of the choice problem, denoted by $\rho$, is given by the full specification of this PI. The decision model over $O$ given $\rho$ is defined by $b \succ_{\rho} c$ iff $\sum_{b \succ_{i} c} W_{i}>\sum_{c \succ_{i} b} W_{i}$.

Definition 1. An option $a \in O$ is called weighted Condorcet winner w.r.t. $\rho$ (noted $\mathrm{WCW}(\rho))$ if for all $b \in O^{\star}:=O \backslash\{a\}, a \succ_{\rho} b$.

We shall also assume throughout this paper the existence of a weighted Condorcet winner labeled $a \in O$. 


\subsection{Description of the language for the explanation}

Following the example in the introduction, the simplest language on the partial preferences is composed of terms of the form $[i: b \succ c]$, with $i \in H$ and $b, c \in O$, meaning that $b$ is strictly preferred to $c$ on criterion $i$. Such terms are called basic preference statements. In order to reduce the length of the explanation, they can also be factored into terms of the form $[I: b \succ P]$, with $I \subseteq H, b \in O$ and $P \subseteq O \backslash\{b\}$, meaning that $b$ is strictly preferred to all options in $P$ on all criteria in $I$. Such terms are called factored preference statements. The set of all subsets of basic preference statements (resp. factored preference statements) that correspond to a total order over $O$ on each criterion is denoted by $\mathcal{S}$ (resp. $\widehat{\mathcal{S}}$ ). For $K \in \mathcal{S}$, we denote by $K^{\uparrow}$ the set of statements of the form $[I: b \succ P]$ with $I \subseteq H$ and $P \subseteq O$ such that for all $i \in I$ and $c \in P,[i: b \succ c] \in K$. Conversely, for $\widehat{K} \in \widehat{\mathcal{S}}$, let $\widehat{K}^{\downarrow}=\{[i: b \succ c]: \exists[I: b \succ P] \in \widehat{K}$ s.t. $i \in I$ and $c \in P\}$ be the atomization of the factored statements $\widehat{K}$. Now assuming that $a$ is the WCW, it is useful to distinguish different types of statements:

- positive statements, of the form $[I: a \succ P]$

- neutral statements, of the form $[I: b \succ P]$ with $a \notin P$

- negative statements, of the form $[I: b \succ P]$ with $a \in P$.

We note that in the case of basic statements, negative statements are "purely" negative since $P=\{a\}$.

Example 2. The full ranking of actions, on criterion 1 only, yields the following basic statements:

- $[1: c \succ a]$ (negative statement),

$-[1: c \succ e],[1: c \succ d],[1: c \succ b],[1: c \succ f],[1: e \succ d],[1: e \succ b,[1: e \succ$ $f],[1: d \succ b],[1: d \succ f],[1: b \succ f]$ (neutral statements),

- $[1: a \succ e],[1: a \succ d],[1: a \succ b],[1: a \succ f]$ (positive statements).

Regarding factored statements, the following examples can be given:

- $[1,2: e \succ d]$ is a neutral statement;

- $[1: c \succ a, e]$ is a negative statement;

- $[1,2: a \succ d, e, f]$ is a positive statement.

The explanation shall also mention the weights in order to be complete. We assume throughout this paper that the values of weights can be shown to the audience. This is obvious in voting committee where the weights are public. This is also a reasonable assumption in a multi-criteria context when the weights are elicited, as the constructed weights are validated by the decision-maker and then become an important element of the explanation [6]. The corresponding language on the weights is simply composed of statements (called importance statements) of the form $[i: \alpha]$ with $i \in H$ and $\alpha \in[0,1]$ meaning that the weight of criterion $i$ is $\alpha$. Let $\mathcal{W}$ (the set of normalized weights) be the set of sets $\left\{\left[i: w_{i}\right]: i \in H\right\}$ such that $w \in[0,1]^{H}$ satisfies $\sum_{i \in H} w_{i}=1$. For $W \in \mathcal{W}$ and $i \in H, W_{i} \in[0,1]$ is the value of the weight on criterion $i$, that is that $\left[i: W_{i}\right] \in W$. A set $A \subseteq H$ is called a winning coalition if $\sum_{i \in A} W_{i}>\frac{1}{2}$. 


\subsection{Cost function over the explanations}

An explanation is a pair composed of an element of $\widehat{\mathcal{S}}$ (note that $\mathcal{S} \subset \widehat{\mathcal{S}}$ ) and an element of $\mathcal{W}$. We seek for minimal explanations in the sense of some cost function. For simplicity, the cost of an element of $\widehat{\mathcal{S}}$ or $\mathcal{W}$ is assumed to be the sum of the cost of its statements. A difficult issue then arises: how should we define the cost of a statement?

Intuitively, the cost should capture the simplicity of the statement, the easiness for the user to understand it. Of course this cost must depend in the end of the basic pieces of information transmitted by the statement. The statements are of various complexity. For instance $[1,2,5,7,9: a \succ b, c, g, h]$ looks more complex to grasp than $[1: a \succ b]$, so that factored preference statements are basically more complex than basic preference statements.

Let us considered the case of preference statements. At this point we make the following assumptions:

- neutrality - the cost is insensitive to the identity of both criteria and options, i.e. $\operatorname{cost}([I: b \succ P])$ depends only on $|I|$ and $|P|$ and is noted $C(|I|,|P|)$,

- monotony - the cost of a statement is monotonic w.r.t. criteria and to options, i.e. function $C$ is non-decreasing in its two arguments. Neutrality implies that all basic statements have the same cost $C(1,1)$.

Additionally to the previous properties, the cost may be sub-additive in the sense that $\operatorname{cost}\left(I \cup I^{\prime}, P\right) \leq \operatorname{cost}(I, P)+\operatorname{cost}\left(I^{\prime}, P\right)$ and $\operatorname{cost}\left(I, P \cup P^{\prime}\right) \leq \operatorname{cost}(I, P)+$ $\operatorname{cost}\left(I, P^{\prime}\right)$, or super-additive if the converse inequalities hold. Finally, we assume the cost function can be computed in polynomial time.

\section{$3 \quad$ Minimal complete explanations}

Suppose now that the PI of choice problem is expressed in the basic language as a pair $\langle S, W\rangle \in \mathcal{S} \times \mathcal{W}$. Explaining why $a$ is the Condorcet winner for $\langle S, W\rangle$ amounts to simplifying the PI (data-based approach [5]). We focus in this section on explanations in the language $\mathcal{S} \times \mathcal{W}$. The case of the other languages will be considered later in the paper.

A subset $\langle K, L\rangle$ of $\langle S, W\rangle$ is called a complete explanation if the decision remains unchanged regardless of how $\langle K, L\rangle$ is completed to form an element of $\mathcal{S} \times \mathcal{W}$. The completeness of the explanation is thus ensured. The pairs are equipped with the ordering $\langle K, L\rangle \sqsubseteq\left\langle K^{\prime}, L^{\prime}\right\rangle$ if $K \subseteq K^{\prime}$ and $L \subseteq L^{\prime}$. More formally, we introduce the next definition.

Definition 2. The set of complete explanations for language $\mathcal{S} \times \mathcal{W}$ is:

$$
\begin{aligned}
& E x_{\mathcal{S}, \mathcal{W}}:=\{\langle K, L\rangle \sqsubseteq\langle S, W\rangle: \\
&\left.\forall K^{\prime} \in \mathcal{S}(K) \forall L^{\prime} \in \mathcal{W}(L) \quad \operatorname{WCW}\left(K^{\prime}, L^{\prime}\right)=\{a\}\right\},
\end{aligned}
$$

where $\mathcal{S}(K)=\left\{K^{\prime} \in \mathcal{S}: K^{\prime} \supseteq K\right\}$ and $\mathcal{W}(L)=\left\{L^{\prime} \in \mathcal{W}: L^{\prime} \supseteq L\right\}$. 
Example 3. The explanation $K_{1}=[1,2: a \succ d, e, f],[1,3: a \succ b],[2,3: a \succ c]$ is not complete, since it does not provide enough evidence that $a$ is preferred over $c$. Indeed, $H_{K_{1}}(a, c)<0$ (since $0.42-0.58=-0.16$ ). On the other hand, $[1: a \succ e, d, b, f],[2: a \succ f, e, d, c],[3: a \succ b, c, d],[4: a \succ c, e],[5: a \succ c]$ is complete but certainly not minimal, since (for instance) exactly the same explanation without the last statement is also a complete explanation whose cost is certainly lower (by monotonicity of the cost function). Now if the cost function is sub-additive, then a minimal explanation cannot contain (for instance) both $[1,2: a \succ d, e]$ and $[1,2: a \succ f]$. This is so because then it would be possible to factor these statements as $[1,2: a \succ d, e, f]$, all other things being equal, so as to obtain a new explanation with a lower cost.

In the rest of the paper, complete explanations will be called simply explanations when there is no possible confusion. One has $\langle S, W\rangle \in E x_{\mathcal{S}, \mathcal{W}}$ and $\langle\emptyset, \emptyset\rangle \notin E x_{\mathcal{S}, \mathcal{W}}$. As shown below, adding more information to a complete explanation also yields a complete explanation.

Lemma 1. If $\langle K, L\rangle \in E x_{\mathcal{S}, \mathcal{W}}$ then $\left\langle K^{\prime}, L^{\prime}\right\rangle \in E x_{\mathcal{S}, \mathcal{W}}$ for all $K^{\prime}, L^{\prime}$ with $K \subseteq$ $K^{\prime} \subseteq S$ and $L \subseteq L^{\prime} \subseteq W$.

Proof : Clear since $\mathcal{S}(K) \supseteq \mathcal{S}\left(K^{\prime}\right)$ when $K \subseteq K^{\prime}$, and $\mathcal{W}(L) \supseteq \mathcal{W}\left(L^{\prime}\right)$ when $L \subseteq L^{\prime}$.

We will assume in the rest of the paper that there is no simplification regarding the preferential information $W$. Indeed the gain of displaying less values of the weights is much less significant than the gain concerning $S$. This comes from the fact that $|W|=m$ whereas $|S|=\frac{1}{2} m p(p-1)$, where $m=|H|$ and $p=|O|$. Only the information about the basic statements $S \in \mathcal{S}$ is simplified. We are thus interested in the elements of $E x_{\mathcal{S}, \mathcal{W}}$ of the form $\langle K, W\rangle$. Hence we introduce the notation $E x_{\mathcal{S}}=\left\{K \in \mathcal{S}:\langle K, W\rangle \in E x_{\mathcal{S}, \mathcal{W}}\right\}$.

\section{Simple language for $S$}

We consider in this section explanations with the basic languages $\mathcal{S}$ and $\mathcal{W}$. In this section, the PI is expressed as $\langle S, W\rangle$. The aim of this section is to characterize and construct minimal elements of $E x_{\mathcal{S}}$ w.r.t. the cost.

We set $H_{K}(a, b):=\sum_{i:[i: a \succ b] \in K} W_{i}-\sum_{i:[i: a \succ b] \notin K} W_{i}$ for $K \subseteq S$ and $b \in O^{\star}$. This means that $K \subseteq S$ is completed only with negative preference statements (in other words, what is not explicitly provided in the explanation is assumed to be negative).

Lemma 2. $E x_{\mathcal{S}}=\left\{K \subseteq S: \forall b \in O^{\star} \quad H_{K}(a, b)>0\right\}$. 
Proof : We have WCW $\left(K^{\prime}, W\right)=\{a\} \forall K^{\prime} \in \mathcal{S}(K)$ iff WCW $\left(K^{\prime}, W\right)=\{a\}$ for $K^{\prime}=K \cup\left\{[i: b \succ a]: b \in O^{\star}\right.$ and $\left.[i: a \succ b],[i: b \succ a] \notin K\right\}$ iff $H_{K}(a, b)>0$ $\forall b \in O^{\star}$.

A consequence of this result is that neutral statements can simply be ignored since they do not affect the expression $H_{K}(a, b)$. The next lemma shows furthermore that the minimal explanations are free of negative statements.

Lemma 3. Let $K \in E x_{\mathcal{S}}$ minimal w.r.t. the cost. Then $K$ does not contain any negative or neutral preference statement.

Proof : $K \in E x_{\mathcal{S}}$ cannot minimize the cost if $[i: b \succ a] \in K$ since then $H_{K^{\prime}}(a, b)=H_{K}(a, b)$ and thus $K^{\prime} \in E x_{\mathcal{S}}$, with $K^{\prime}=K \backslash\{[i: b \succ a]\}$. It is the same if $[i: b \succ c] \in K$ with $b, c \neq a$.

Then we prove that we can replace a positive basic statement appearing in a complete explanation by another one, while having still a complete explanation, if the weight of the criterion involved in the first statement is not larger than that involved in the second one.

Lemma 4. Let $K \in E x_{\mathcal{S}},[i: a \succ b] \in K$ and $[j: a \succ b] \in S \backslash K$ with $W_{j} \geq W_{i}$. Then $(K \backslash\{[i: a \succ b]\}) \cup\{[j: a \succ b]\} \in E x_{\mathcal{S}}$.

Proof : Let $K^{\prime}=(K \backslash\{[i: a \succ b]\}) \cup\{[j: a \succ b]\}$. We have $H_{K^{\prime}}(a, b)=$ $H_{K}(a, b)+2\left(W_{j}-W_{i}\right)>0$. Hence $K^{\prime} \in E x_{\mathcal{S}}$.

We define $\Delta_{i}^{S}(a, b)=+1$ if $[i: a \succ b] \in S$, and $\Delta_{i}^{S}(a, b)=-1$ if $[i: b \succ a] \in$ $S$. For each option $b \in O^{\star}$, we sort the criteria in $H$ by a permutation $\pi_{b}$ on $H$ such that $W_{\pi_{b}(1)} \Delta_{\pi_{b}(1)}^{S}(a, b) \geq \cdots \geq W_{\pi_{b}(m)} \Delta_{\pi_{b}(m)}^{S}(a, b)$.

Proposition 1. For each $b \in O^{\star}$, let $p_{b}$ the smallest integer such that $H_{K_{p_{b}}^{b}}(a, b)>0$, where $K_{p_{b}}^{b}=\left\{\left[\pi_{b}(1): a \succ b\right],\left[\pi_{b}(2): a \succ b\right], \ldots,\left[\pi_{b}\left(p_{b}\right):\right.\right.$ $a \succ b]\}$. Then $\left\{\left[\pi_{b}(j): a \succ b\right]: b \in O^{\star}\right.$ and $\left.j \in\left\{1, \ldots, p_{b}\right\}\right\}$ is a minimal element of $E x_{\mathcal{S}}$ w.r.t. the cost.

Proof (Sketch): Let $E x_{\mathcal{S}}(b)=\left\{K \subseteq S_{b}: H_{K}(a, b)>0\right\}$, where $S_{b}$ is the set of statements of $S$ involving option $b$. The existence of $p_{b}$ follows from the fact that $a$ is a WCW. Now let $j \in\left\{1, \ldots, p_{b}-1\right\}$. From the definition of $p_{b}$, $K_{p_{b}-1}^{b} \notin E x_{\mathcal{S}}(b)$. This, together with $W_{\pi_{b}(j)} \geq W_{\pi_{b}\left(p_{b}\right)}$ and Lemma 4, implies that $K_{p_{b}}^{b} \backslash\left\{\left[\pi_{b}(j): a \succ b\right]\right\} \notin E x_{\mathcal{S}}(b)$. Hence $K_{p_{b}}^{b}$ is minimal in $E x_{\mathcal{S}}(b)$ in the sense of $\subseteq$. It is also apparent from Lemma 4 that there is no element of $E x_{\mathcal{S}}(b)$ with a strictly lower cardinality and thus lower cost (since, from Section 2.3, the cost of a set of basic statements is proportional to its cardinality). Finally, $\cup_{b \in O^{\star}} K_{p_{b}}$ minimizes the cost in $E x_{\mathcal{S}}$ since the conditions on each option $b \in O^{\star}$ are independent.

This proposition provides a polynomial computation of a minimal element of $E x_{\mathcal{S}}$. This is obtained for instance by the following greedy Algorithm 1. The complexity of this algorithm is $O(m \cdot p \cdot \log (p))$ (where $m=|H|$ and $p=|O|$ ). 


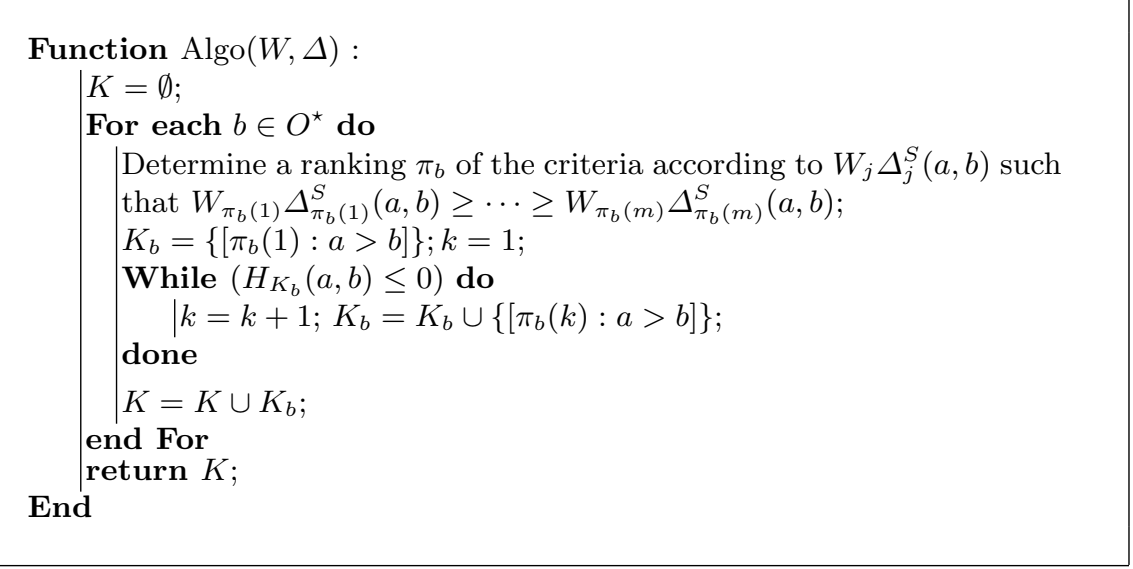

Algorithm 1: Algorithm for the determination of a minimal element of $E x_{\mathcal{S}}$. The outcome is $K$.

We illustrate this on our example.

Example 4. Consider the iteration regarding option $b$. The ranking of criteria

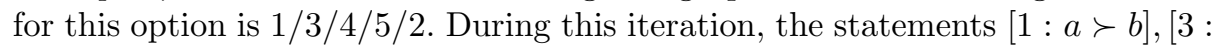
$a \succ b]$ are added to the explanation. In the end the explanation produced by Algorithm 1 is $[1: a \succ b],[3: a \succ b],[2: a \succ c],[3: a \succ c],[4: a \succ c],[1: a \succ$ $d],[2: a \succ d],[1: a \succ e],[2: a \succ e],[1: a \succ f],[2: a \succ f]$. Note that criterion 5 is never involved in the explanation.

\section{$5 \quad$ Factored language for $S$}

The language used in the previous section is simple but not very intuitive. As illustrated in the introduction, a natural extension is to allow more compact explanations by means of factored statements. We thus consider in this section explanations with the factored language $\widehat{\mathcal{S}}$ and the basic language $\mathcal{W}$. As in previous section, all weight statements in $W \in \mathcal{W}$ are kept. The explanations for $\widehat{\mathcal{S}}$ are:

$$
E x_{\widehat{\mathcal{S}}}=\left\{\widehat{K} \subseteq S^{\uparrow}: \forall K \in \mathcal{S}\left(\widehat{K}^{\downarrow}\right) \quad \mathrm{WCW}(K, W)=\{a\}\right\} .
$$

Similarly to what was proved for basic statements, it is simple to show that minimal explanation must only contain positive statements.

Lemma 5. Let $\widehat{K} \in E x_{\widehat{\mathcal{S}}}$ minimal w.r.t. the cost. Then $\widehat{K}$ only contains positive preference statements.

Proof : Similar to the proof of Lemma 3.

A practical consequence of this result is that it is sufficient to represent the PI as a binary matrix, for $a$, where an entry 1 at coordinates $(i, j)$ represents 
the fact that the option $i$ is less preferred than $a$ on criteria $j$. Doing so, we do not encode the preferential information expressed by neutral statements.

This representation is attractive because factored statements visually correspond to (combinatorial) rectangles. Informally, looking for an explanation amounts to find a "cheap" way to "sufficiently" cover the 1's in this matrix. However, an interesting thing to notice is that a minimal explanation with factored statements does not imply that factored statements are non overlapping. To put it differently, it may be the case that some preferential information is repeated in the explanations. Consider the following example:

Example 5. There are 5 criteria of equal weight and 6 options, and $a$ is the weighted Condorcet winner. As for the cost of statements, it is constant whatever the statement.

\begin{tabular}{|c|ccccc|}
\hline & 1 & 2 & 3 & 4 & 5 \\
\hline & 0.2 & 0.2 & 0.2 & 0.2 & 0.2 \\
\hline \hline$b$ & 1 & 1 & 0 & 0 & 1 \\
$c$ & 1 & 1 & 0 & 1 & 0 \\
$d$ & 1 & 1 & 1 & 0 & 0 \\
$e$ & 0 & 1 & 1 & 0 & 1 \\
$f$ & 0 & 1 & 1 & 1 & 0 \\
\hline
\end{tabular}

There are several minimal explanations involving 4 statements, but all of them result in a covering in the matrix, like for instance $[1,2: a \succ b, c, d],[2,3: a \succ$ $d, e, f],[4: a \succ c, f][5: a \succ b, e]$, where the preferential information that $a \succ_{2} d$ is expressed twice (in the first and second statement).

The previous section concluded on a simple algorithm to compute minimal explanations with basic statements. Unfortunately, we will see that the additional expressive power provided by the factored statements comes at a price when we want to compute minimal explanations.

Proposition 2 (Min. explanations with factored statements). Deciding if (using factored statements $S^{\uparrow}$ ) there exists an explanation of cost at most $k$ is NP-complete. This holds even if criteria are unweighted and if the cost of any statement is a constant.

Proof (Sketch): Membership is direct since computing the cost of an explanation can be done in polynomial time. We show hardness by reduction from the Biclique Edge Cover (BEC), known to be NP-complete (problem [GT18] in $[7])$. In BEC, we are given a finite bipartite graph $G=(X, Y, E)$ and positive integer $k^{\prime}$. A biclique is a complete bipartite subgraph of $G$, i.e., a subgraph induced by a subset of vertices such that any vertex is connected to a vertex of the other part. The question is whether there exists a collection of bicliques covering edges of $G$ of size at most $k^{\prime}$.

Let $I=(X, Y, E)$ be an instance of BEC. From $I$, we build an instance $I^{\prime}$ of the explanation problem as follows. The set $O$ of actions contains $O_{1}=$ $\left\{o_{1}, \ldots, o_{n}\right\}$ corresponding to the elements in $X$, and a set $O_{2}$ of dummy actions consisting of $n+3$ actions $\left\{o_{1}^{\prime}, \ldots, o_{n+3}^{\prime}\right\}$. The set $H$ of criteria contains $H_{1}=$ 
$\left\{h_{1}, \ldots, h_{n}\right\}$ corresponding to the elements in $Y$, and a set $H_{2}$ of dummy criteria consisting of $n+3$ criteria $\left\{h_{1}^{\prime}, \ldots, h_{n+3}^{\prime}\right\}$. First, for each $\left(x_{i}, y_{j}\right) \in E$, we build a statement $\left[h_{i}: a \succ o_{j}\right]$. Let $S_{O_{1}, H_{1}}$ be this set of statements. Observe that a factored statement $[I: a \succ P]$ with $I \subseteq H_{1}$ and $P \subseteq O_{1}$ correspond to a biclique in $I$. But $a$ may not be a Condorcet winner. Thus for each action $o \in O_{1}$, we add $(n+2)-\left|\left\{\left[h_{i}: a \succ o\right] \in O_{1}\right\}\right|$ statement(s) $\left[h_{j}^{\prime}: a \succ o\right]$. Let $S_{O_{1}, H_{2}}$ be this set of statements. Note that at this point, $a$ is preferred to any other $o \in O_{1}$ by $n+2$ criteria. Next $\forall\left(h_{i}^{\prime}, o_{j}^{\prime}\right) \in\left(H_{2} \times O_{2}\right)$ such that $i \neq j$ we add the following statement: $\left[h_{i}^{\prime}: a \succ o_{j}^{\prime}\right]$. There are $n+2$ such statements, hence $a$ is preferred to any other $o \in \mathrm{O}_{2}$ by a majority of exactly $n+2$ criteria. Let $S_{\mathrm{O}_{2}, \mathrm{H}_{2}}$ be this set of statements. We claim that $I$ admits a biclique vertex partition of at most $k-(n+3)$ subsets iff $I^{\prime}$ admits an explanation $\widehat{K}_{*}$ of cost at most $k$ using factored statements. Take $(\Leftarrow)$. By construction, all the basic statements must be "covered", i.e. $\widehat{K}_{*}^{\downarrow}=S_{O_{1}, H_{1}} \cup S_{O_{1}, H_{2}} \cup S_{O_{2}, H_{2}}$. We denote by $\operatorname{cov}($.) the cost of covering a set of basic statements of $S_{O, H}$ (this is just the number of factored statements used, as the cost of statements is constant). Furthermore, as there are no statements using actions from $\mathrm{O}_{2}$ and criteria from $H_{1}$, no factored statement can cover at the same time statements from $S_{O_{1}, H_{1}}$ and $S_{O_{2}, H_{2}}$. Hence $\operatorname{cost}\left(\widehat{K}_{*}\right)=\operatorname{cov}\left(S_{O_{1}, H_{1}} \cup S^{\prime}\right)+\operatorname{cov}\left(S_{O_{2}, H_{2}} \cup S^{\prime \prime}\right)$, such that $S^{\prime} \cup S^{\prime \prime}=S_{O_{1}, H_{2}}$.

But now observe that $\operatorname{cov}\left(S_{O_{2}, H_{2}}\right)=\operatorname{cov}\left(S_{O_{2}, H_{2}} \cup S_{O_{1}, H_{2}}\right)=n+3$, so $\operatorname{cost}\left(\widehat{K}_{*}\right)$ boils down to $n+3+\operatorname{cov}\left(S_{O_{1}, H_{1}} \cup S^{\prime}\right)$. By monotony wrt. criteria, $\operatorname{cov}\left(S_{O_{1}, H_{1}} \cup S^{\prime}\right)$ is minimized when $S^{\prime}=\emptyset$, and this leads to the fact $\operatorname{cov}\left(S_{O_{1}, H_{1}}\right) \leq k-(n+3)$. The $(\Rightarrow)$ direction is easy.

The previous result essentially shows that when the cost function implies to minimize the number of factored statements, no efficient algorithm can determine minimal explanations (unless $\mathrm{P}=\mathrm{NP}$ ). But there may be specific class(es) of cost functions for which the problem may turn out to be easy. As shown in the next lemma, when the cost function is super-additive, then it is sufficient to look for basic statements.

Lemma 6. If the cost function is super-additive, then $\min _{\widehat{K} \in E x_{\widehat{\mathcal{S}}}} \operatorname{cost}(\widehat{K})=$ $\min _{K \in E x_{\mathcal{S}}} \operatorname{cost}(K)$.

Proof : Let $\widehat{K} \in E x_{\widehat{\mathcal{S}}}$. We know that $\widehat{K}^{\downarrow} \in E x_{\mathcal{S}}$. By super-additivity, $\operatorname{cost}(\widehat{K})=\sum_{[I: b \succ P] \in \widehat{K}} \operatorname{cost}([I: b \succ P]) \geq \sum_{[I: b \succ P] \in \widehat{K}} \sum_{i \in I, c \in P} \operatorname{cost}([i: b \succ$ $c]) \geq \sum_{[i: b \succ c] \in \widehat{K}^{\downarrow}} \operatorname{cost}([i: b \succ c])=\operatorname{cost}\left(\widehat{K}^{\downarrow}\right)$.

Yet, the cost is expected to be sub-additive. Relations (1) and (2) below give examples of sub-additive cost functions. In this case, factored statements are less costly (e.g. the cost of $[\{1,2\}: a \succ b]$ should not be larger than the cost of $[1: a \succ b],[2: a \succ b])$ and factored explanations become very relevant.

When the cost function is sub-additive, an intuitive idea could be to restrict our attention to statements which exhibit winning coalitions. For that purpose, let us assign to any subset $P \subseteq O^{\star}$ defended by a winning coalition the cost of using such statement. A practical way to do this is to build $T: 2^{O^{\star}} \rightarrow 2^{H}$ 
such that for all subsets $P \subseteq O^{\star}, T(P)$ is the largest set of criteria for which $[T(P): a \succ P] \in S^{\uparrow}$. We have $T(P)=\cap_{b \in P} T(\{b\})$, where $T(\{b\}):=\{i \in H:$ $[i: a \succ b] \in S\}$. Then subsets $P$ of increasing cardinality are considered (but those supported by non-winning coalitions are discarded). The cost $C(\alpha,|P|)$ is finally assigned, where $\alpha$ is the size of the smallest winning coalition contained in $T(P)$. Then, the problem can be turned into a weighted set packing, for which the direct ILP formulation would certainly be sufficient in practice for reasonable values of $|O|$ and $|H|$.

Example 6. On our running example, the different potential factors would be $T(\{b\})=\{1,3\}$ with $C(2,1), T(\{c\})=\{2,3,4,5\}$ with $C(4,1), T(\{d\})=$ $\{1,2,3\}$ with $C(3,1), T(\{e\})=\{1,2,4\}$ with $C(3,1), T(\{f\})=\{1,2\}$ with $C(2,1), T(\{b, d\})=\{1,3\}$ with $C(2,2)$, etc. Depending on the cost function, two possible explanations remain: $\widehat{K}_{1}=\{[1,3: a \succ b],[2,3,4,5: a \succ c],[1,2$ : $a \succ d, e, f]\}$ for a cost of $C(2,1)+C(4,1)+C(2,3)$, and $\widehat{K}_{2}=\{[1,3: a \succ$ $b, d],[2,3,4,5: a \succ c],[1,2: a \succ e, f]$ for a cost of $C(2,2)+C(4,1)+C(2,2)$. The cost function

$$
C(i, j)=i^{\alpha} j^{\beta}
$$

(which is sub-additive when $\alpha \leq 1$ and $\beta \leq 1$ ) would select $\widehat{K}_{1}$. Note that criteria 4 or 5 will be dropped from the statement $[T(\{c\}): a \succ c]$.

Now, considering only factored statements with winning coalitions may certainly prevent from reaching optimal factored explanations, as we illustrate below.

Example \%. We have 4 criteria and 3 options. Assume that $a$ is preferred to $b$ on criteria 1,2 , and 3 ; that $a$ is preferred to $c$ on criteria 1,2 , and 4 and that any coalition of at least 3 criteria is winning. The previous approach based on $T$ gives $\widehat{K}_{1}=\{[1,2,3: a \succ b],[1,2,4: a \succ c]\}$, with $\operatorname{cost}\left(\widehat{K}_{1}\right)=2 C(3,1)$. Algorithm 1 gives $\widehat{K}_{2}=\left(\widehat{K}_{1}\right)^{\downarrow}$ with $\operatorname{cost}\left(\widehat{K}_{2}\right)=6 C(1,1)$. Another option is to consider $\widehat{K}_{3}=\{[1,2: a \succ b, c],[3: a \succ b][4: a \succ c]\}$, with $\operatorname{cost}\left(\widehat{K}_{3}\right)=C(2,2)+2 C(1,1)$. Let us consider the following cost function ${ }^{4}$

$$
C(i, j)=i \log (j+1) .
$$

Function $C$ is sub-additive, since $C\left(i+i^{\prime}, j\right)=C(i, j)+C\left(i^{\prime}, j\right)$ and, from relation $j+j^{\prime}+1 \leq(j+1)\left(j^{\prime}+1\right)$, we obtain $C\left(i, j+j^{\prime}\right) \leq C(i, j)+C\left(i, j^{\prime}\right)$. Then we have $\operatorname{cost}\left(\widehat{\widehat{K}}_{3}\right)<\operatorname{cost}\left(\widehat{K}_{1}\right)=\operatorname{cost}\left(\widehat{K}_{2}\right)$ so that the explanation with the smallest cost is $\widehat{K}_{3}$.

Enforcing complete explanations implies a relatively large number of terms in the explanation. However, in most cases, factored statements allow to obtain small explanations. For instance, when all criteria have the same weight, the minimal elements of $E x_{\mathcal{S}}$ contain exactly $(p-1) n$ basic statements (where $p=$

\footnotetext{
${ }^{4}$ capturing that factoring over the criteria is more difficult to handle than factoring over the options.
} 
$|O|, m=|H|$ and $m=2 n-1$ if $m$ is odd, and $m=2 n-2$ if $m$ is even. Indeed, one needs $p-1$ terms to explain that $a$ is globally preferred over $b$, for all $b \in O^{\star}$, and the minimal elements of $E x_{\widehat{\mathcal{S}}}$ contain at most $p-1$ factored statements (factoring with winning coalitions for each $b \in O^{\star}$ ).

A current matter of investigation is to determine the class of cost functions for which the minimal explanation is not given either by trivial atomization or by factoring with winning coalitions only, thus requiring dedicated algorithms.

\section{Related Work and Conclusion}

The problem of producing explanations for complex decisions is a long-standing issue in Artificial Intelligence in general. To start with, it is sometimes necessary to (naturally) explain that no satisfying option can be found because the problem is over-constrained $[8,9]$. But of course it is also important to justify why an option is selected among many other competing options, as is typically the case in recommendations. Explanations based on the data seek to focus on a small subpart of the data, sufficient to either convince or indeed prove the claim to the user. Depending on the underlying decision model, this can turn out to be very challenging.

In this paper we investigate the problem of providing minimal and complete explanations for decisions based on a weighted majority principle, when a Condorcet winner exists. A first contribution of this paper is to set up the framework allowing to analyze notions of minimal explanations, introducing in particular different languages to express the preferential information. We then characterize minimal explanations, and study their computational properties. Essentially, we see that producing minimal explanations is easy with basic statements but may be challenging with more expressive languages.

Much work in argumentation set up theoretical systems upon which various types of reasoning can be performed, in particular argument-based decisionmaking has been advocated in [10]. The perspective taken in this paper is different in at least two respects: (i) the decision model is not argumentative in itself, the purpose being instead to generate arguments explaining a multiattribute decision model (weighted majority) issued from decision-theory; and (ii) the arguments we produce are complete (so, really proving the claim), whereas in argumentation the defeasible nature of the evidence put forward is a core assumption [11]. Regarding (ii), our focus on complete arguments has been justified in the introduction. Regarding (i), we should emphasize that we make no claim on the relative merits of argument-based vs. decision-theoretic models. But in many organizations, these decision models are currently in use, and although it may be difficult to change the habits of decision-makers for a fully different approach, adding explanatory features on top on their favorite model can certainly bring much added-value. This approach is not completely new, but previous proposals are mainly heuristic and seek to generate natural arguments [1] that are persuasive in practice. An exception is the recent proposal of [6] which provides solid theoretical foundations to produce explanations for a range 
of decision-theoretic weight-based models, but differs in (ii) since explanations are based on (defeasible) argument schemes. Our focus on complete explanations is a further motivation to build on solid theoretical grounds (even though weaker incomplete arguments may prove more persuasive in practice).

Recently, the field of computational social choice has emerged at the interface of AI and social choice, the study of various computational of various voting systems being one of the main topic in this field. There are connections to our work (and indeed one of the motivating example is a voting committee): for instance, exhibiting the smallest subsets of votes such that a candidate is a necessary winner [12] may be interpret as a minimal (complete) explanation that this candidate indeed wins. However, the typical setting of voting (e.g. guaranteeing the anonymity of voters) would not necessarily allow such explanations to be produced, as it implies to identify voters (to assign weights). An interesting avenue for future research would be to investigate what type of explanations would be acceptable in this context, perhaps balancing the requirements of privacy and the need to support the result. We believe our approach could be relevant. Indeed, two things are noteworthy: first, the proposed approach already preserves some privacy, since typically only parts of the ballots need to be exhibited. Secondly, in many cases it would not be necessary to exactly identify voters, at least when their weights are sufficiently close. Take again our running example: to explain that $a$ beats $b$ we may well say "the most important voter 1 is for $a$, and among 2 and 3 only one defends $b "$.

We conclude by citing some possible extensions of this work. The first is to improve further the language used for explanations. The limitations of factored statements is clear when the following example is considered:

Example 8. In the following example with 6 alternatives and 5 criteria (with the same weight), the factored statements present in any minimal explanation contain at least 3 criteria or alternatives (for instance, $[1,2,3: a \succ e, f],[3,4,5$ : $a \succ b, c],[1,2,4: a \succ d])$

\begin{tabular}{|ccccc|}
\hline 1 & 2 & 3 & 4 & 5 \\
\hline 0.2 & 0.2 & 0.2 & 0.2 & 0.2 \\
\hline$b$ & $c$ & $d$ & $e$ & $f$ \\
$a$ & $a$ & $a$ & $a$ & $a$ \\
$c$ & $d$ & $e$ & $f$ & $b$ \\
$d$ & $e$ & $f$ & $b$ & $c$ \\
$e$ & $f$ & $b$ & $c$ & $d$ \\
$f$ & $b$ & $c$ & $d$ & $e$ \\
\hline
\end{tabular}

However, an intuitive explanation that comes directly to mind is as follows: " $a$ is only beaten by a different option on each criteria".

To take a step in the direction of such more natural explanations, the use of "except" statements allowing to assert that an option is preferred over any other option except the ones explicitly cited should be taken into account. (In fact, the informal explanation of our example makes also use of such a statement, since it essentially says that $a$ is better than $c$ on all criteria except 1 ). In that case, 
minimal explanations may cover larger sets of basic statements than strictly necessary (since including more elements of the PI may allow to make use of an except statement). Another extension would be to relax the assumption of neutrality of the cost function, to account for situations where some information is exogenously provided regarding criteria to be used preferably in the explanation (this may be based on the profile of the decision-maker, which may be more sensible to certain types of criteria).

Acknowledgments. We would like to thank Yann Chevaleyre for discussions related to the topic of this paper. The second author is partly supported by the ANR project ComSoc (ANR-09-BLAN-0305).

\section{References}

1. Carenini, G., Moore, J.: Generating and evaluating evaluative arguments. Artificial Intelligence 170 (2006) 925-952

2. Klein, D.: Decision analytic intelligent systems: automated explanation and knowledge acquisition. Lawrence Erlbaum Associates (1994)

3. Buchanan, B.G., Shortliffe, E.H.: Rule Based Expert Systems: The Mycin Experiments of the Stanford Heuristic Programming Project. Addison-Wesley, Boston, MA, USA (1984)

4. Symeonidis, P., Nanopoulos, A., Manolopoulos, Y.: MoviExplain: a recommender system with explanations. In: Proceedings of the third ACM conference on Recommender systems (RecSys'09), New York, NY, USA, ACM (2009) 317-320

5. Herlocker, J.L., Konstan, J.A., Riedl, J.: Explaining collaborative filtering recommendations. In: Proceedings of the ACM conference on Computer supported cooperative work (CSCW'00), New York, NY, USA, ACM (2000) 241-250

6. Labreuche, C.: A general framework for explaining the results of a multi-attribute preference model. Artificial Intelligence 175 (2011) 1410-1448

7. Garey, M., Johnson, D.: Computers and intractability. A guide to the theory of NP-completeness. Freeman (1979)

8. Junker, U.: QUICKXPLAIN: Preferred explanations and relaxations for overconstrained problems. In McGuinness, D.L., Ferguson, G., eds.: Proceedings of the Nineteenth AAAI Conference on Artificial Intelligence (AAAI'04), AAAI Press (2004) 167-172

9. O'Sullivan, B., Papadopoulos, A., Faltings, B., Pu, P.: Representative explanations for over-constrained problems. In: Proceedings of the Twenty-Second AAAI Conference on Artificial Intelligence (AAAI'07), AAAI Press (2007) 323-328

10. Amgoud, L., Prade, H.: Using arguments for making and explaining decisions. Artificial Intelligence 173 (2009) 413-436

11. Loui, R.P.: Process and policy: Resource-bounded nondemonstrative reasoning. Computational Intelligence 14(1) (1998) 1-38

12. Konczak, K., Lang, J.: Voting procedures with incomplete preferences. In Brafman, R., Junker, U., eds.: Proceedings of the IJCAI-05 Workshop on Advances in Preference Handling. (2005) 124-129 